\title{
Purification of human adipose-derived stem cells from fat tissues using PLGA/silk screen hybrid membranes
}

\begin{abstract}
The purification of human adipose-derived stem cells (hADSCs) from human adipose tissue cells (stromal vascular fraction) was investigated using membrane filtration through poly(lactide-co-glycolic acid)/silk screen hybrid membranes. Membrane filtration methods are attractive in regenerative medicine because they reduce the time required to purify hADSCs (i.e., less than $30 \mathrm{~min}$ ) compared with conventional culture methods, which require $5 \mathrm{II} 12$ days. hADSCs expressing the mesenchymal stem cell markers CD44, CD73, and CD90 were concentrated in the permeation solution from the hybrid membranes. Expression of the surface markers CD44, CD73, and CD99 on the cells in the permeation solution from the hybrid membranes, which were obtained using $18 \mathrm{~mL}$ of feed solution containing $50 \times 104$ cells, was statistically significantly higher than that of the primary adipose tissue cells, indicating that the hADSCs can be purified in the permeation solution by the membrane filtration method. Cells expressing the stem cell-associated marker CD34 could be successfully isolated in the permeation solution, whereas CD34+ cells could not be purified by the conventional culture method. The hADSCs in the permeation solution demonstrated a superior capacity for osteogenic differentiation based on their alkali phosphatase activity, their osterix gene expression, and the results of mineralization analysis by Alizarin Red $\mathrm{S}$ and von Kossa staining compared with the cells from the suspension of human adipose tissue. These results suggest that the hADSCs capable of osteogenic differentiation preferentially permeate through the hybrid membranes.
\end{abstract}

Keyword: Adipose-derived stem cells; Bioseparation; Poly(lactide-co-glycolic acid; Differentiation; Osteoblast; Membrane purification 